\title{
ACCEPTANCE AVAILABILITY OF AGRICULTURAL KNOWLEDGE SYSTEM ACTORS IN EL-DAKAHLIA GOVERNORATE FOR INTEGRATION AND COORDINATION ASSUMPTIONS \& ITS LOCAL APPLY OPPORTUNITIES
}

Zahran, Y. A. ${ }^{(*)}$; E. Yousef ${ }^{(* *)}$; H. S. Kasem ${ }^{(*)}$ and Shimaa M. A. Naba ${ }^{(* *)}$

${ }^{(*)}$ Extension Agricultural and Rural Development Department, Faculty of Agric. - Mansoura University

${ }^{(* *)}$ Extension Agricultural Researches Institute, Agricultural Researches center

\section{مستوى قبول أطراف النظام المعرفى الزراعى بمحافظة الدقهيـة لمفترضـات التكامل والتسـيق وفرص تطبيقها

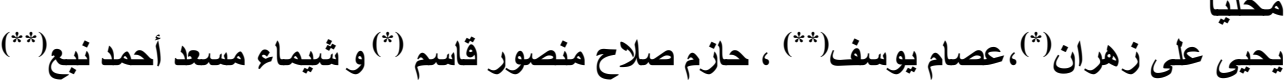

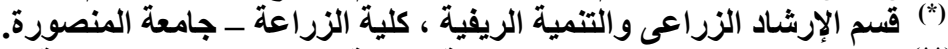

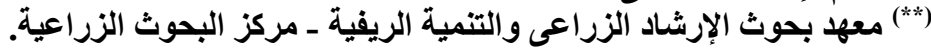 \\ الملخص}

استهدف هذا البحث بصفة رئيسية التعرف على مستوى قبول أطر اف النظام المعرفى الزر اعى بمحافظة الدقهلية لمفترضـات التكامل و التنسيق

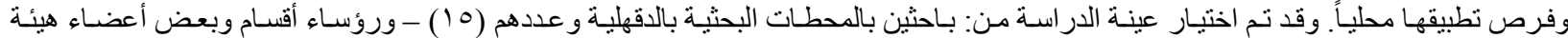

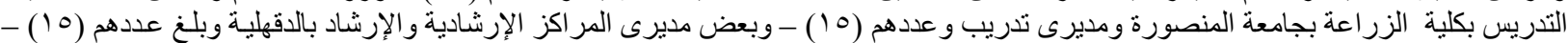

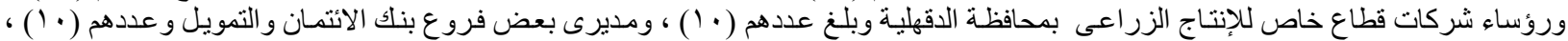

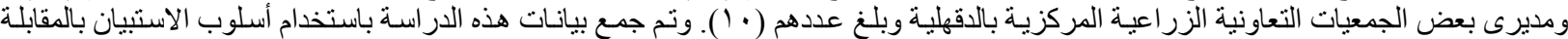

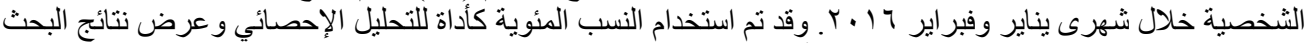

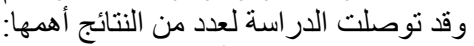

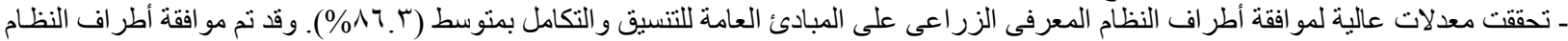

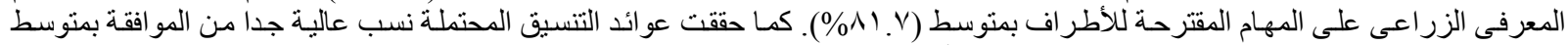

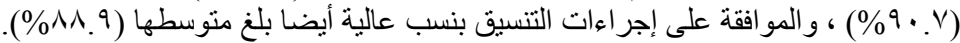

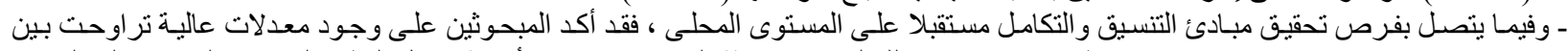

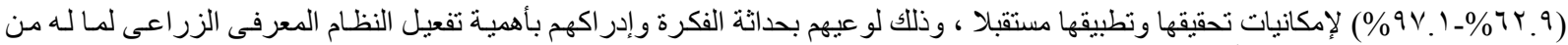
مزايا ومنافع ستعود على كل الأطر اف آن.

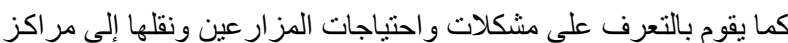

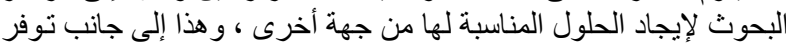

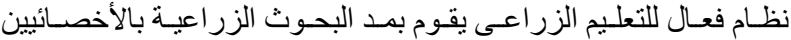

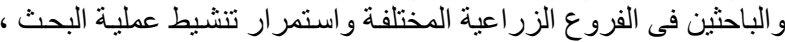

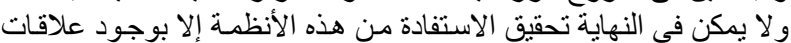

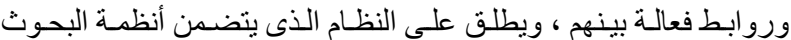

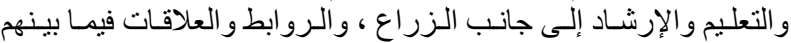

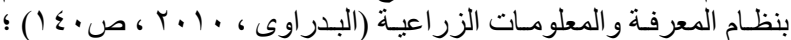

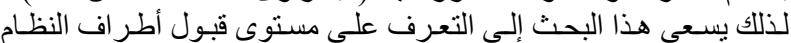

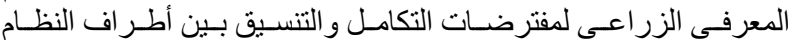

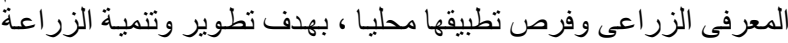

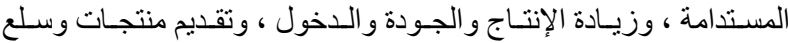
وخدمات مستحدثة وتحسين سبل العيش لمختلف الإنت المستهدوفين.

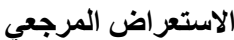
أولا: النظام المعرفى الزراعي المرفى (المفهوم والمهام الرئيسية):

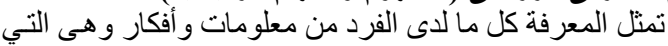

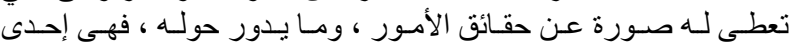

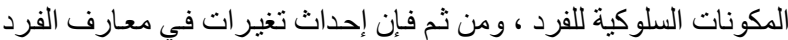

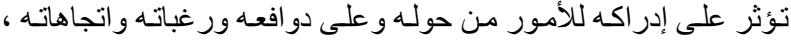
وبطبيعة الحال ينوقف ذلك على الاتجاه الذى تم إحداث التغيير إليه سواء كان ايجابيا أو سلبيا.

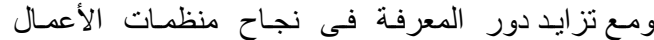

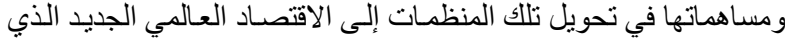

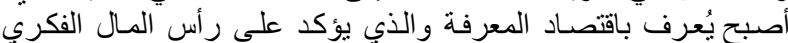

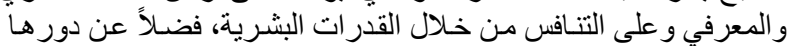

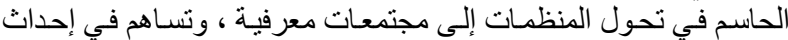
التغيير الجذري في المنظمة لتتكيف مـع التغير السريع في بيئتها. العلى العلى

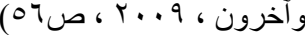

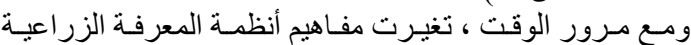
(AKS)

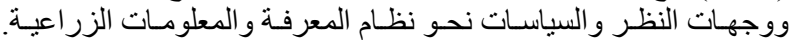
فيرى ( Leeuwis \& Van den Ban, 2004, p.36) أن مفهوم النظام النطام

\section{المقدمة والمشكثة البحثية}

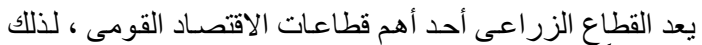

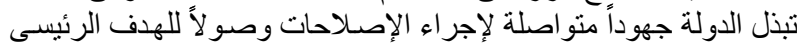

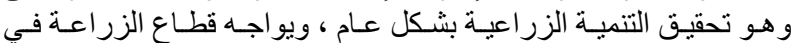

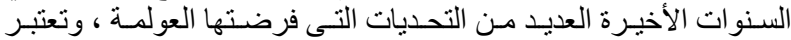

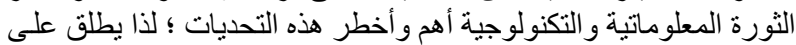

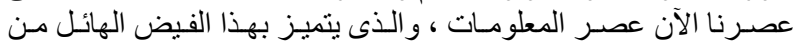

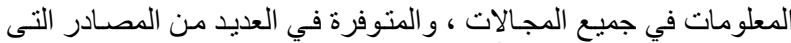

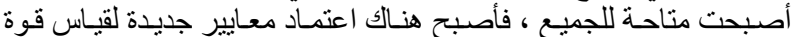

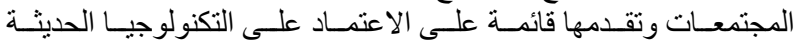

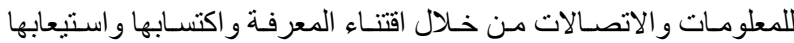

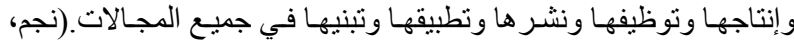

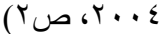

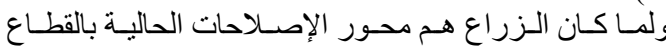

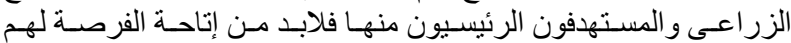

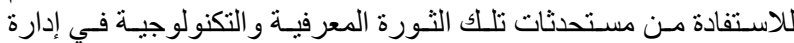

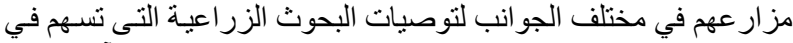

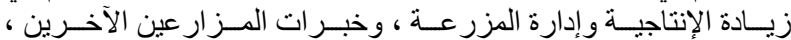

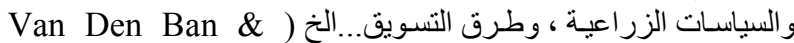
.(Hawkins, 1996, p.25

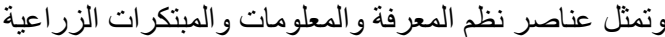

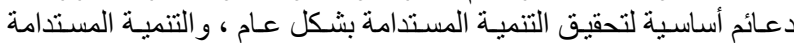

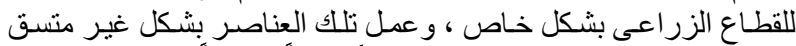

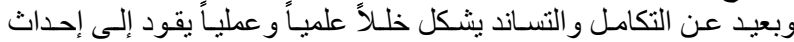

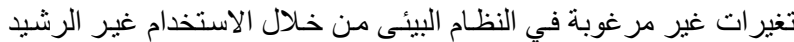

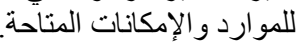

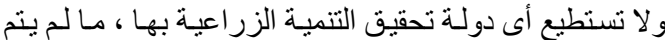

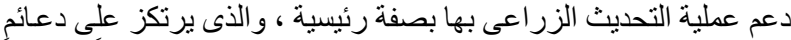

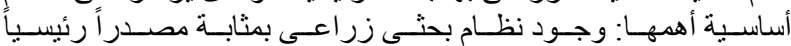

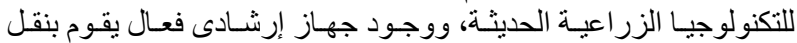

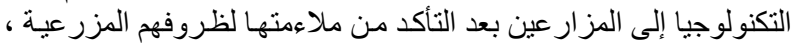


ثانيا: التطور التاريخى لنظم المعرفة والمعلومات الزراعية:

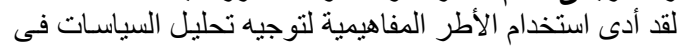

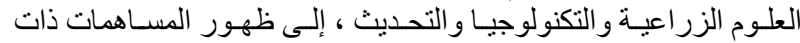

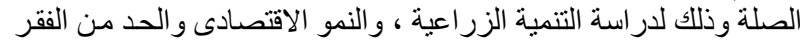
، (Rivera, 2006, p.p.50-60) ، (Chema et al, 2003, p.18)

(World Bank, 2006, p.p.6-7)

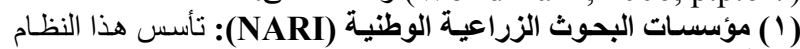

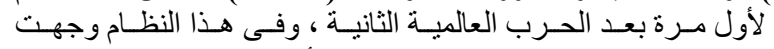

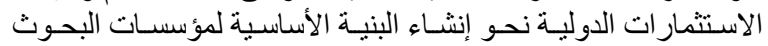

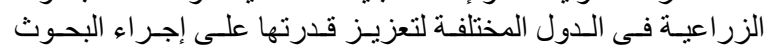

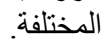

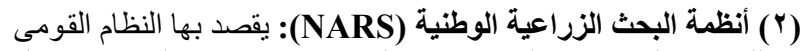

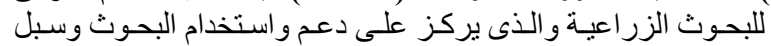

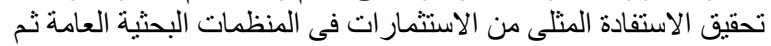

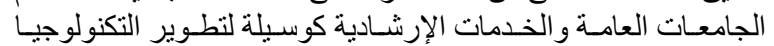

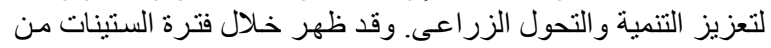

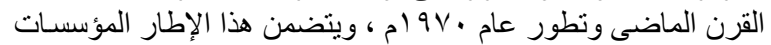

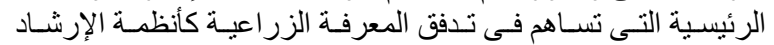

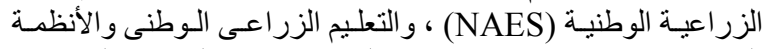

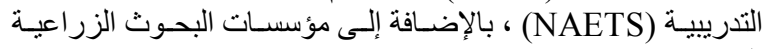

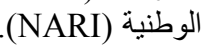

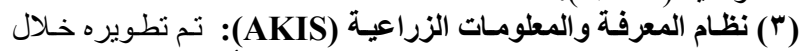

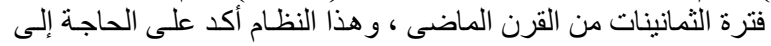

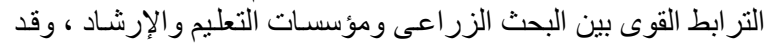

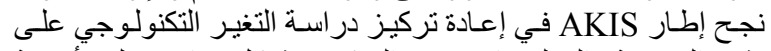

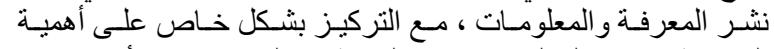

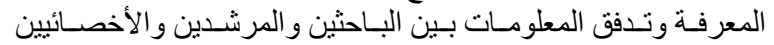

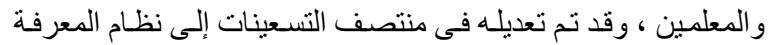

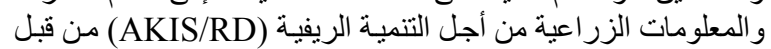

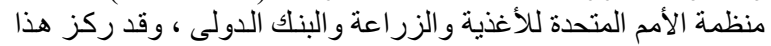

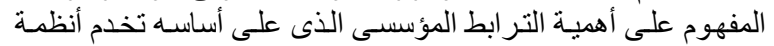
المعرفة التنمية الريفية.

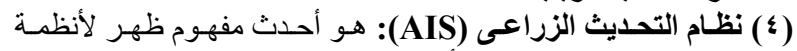

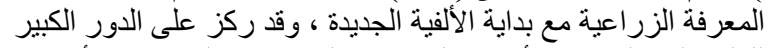

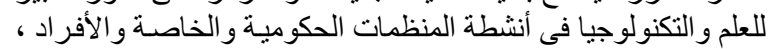

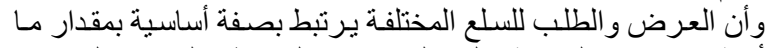

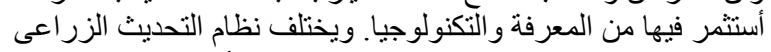

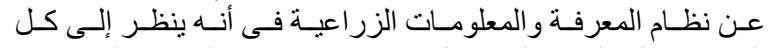

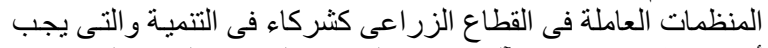

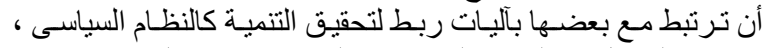

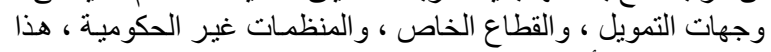

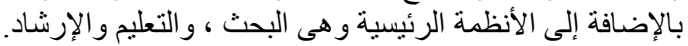

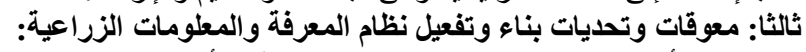

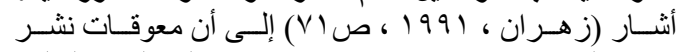

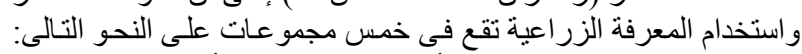

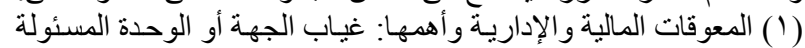

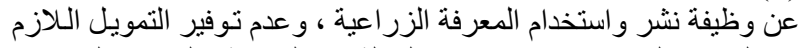

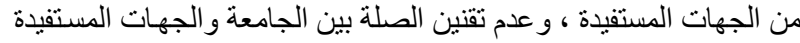

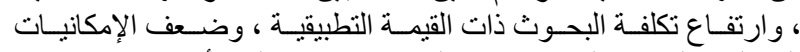

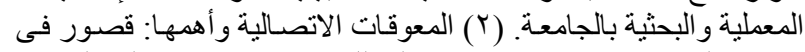

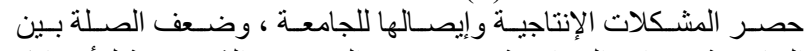

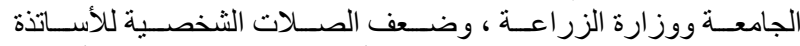

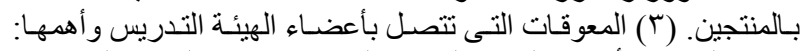

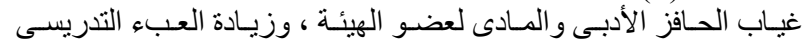

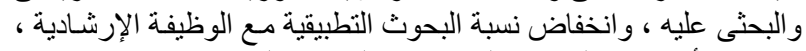

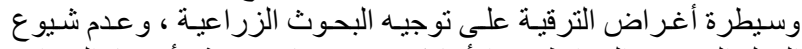

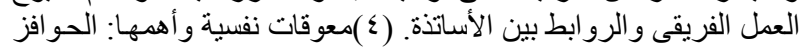

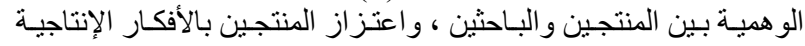

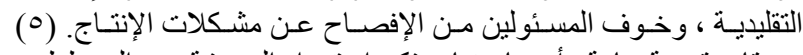

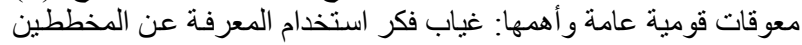

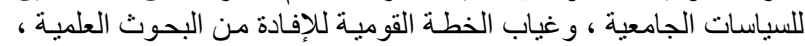
و عدم التنسيق بين الجامعة في هذاب الصغا الصدد.

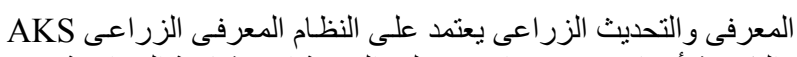

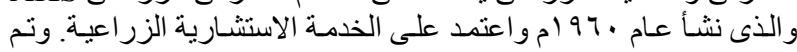

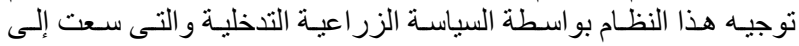

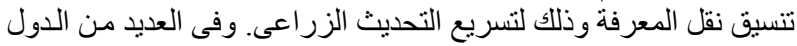

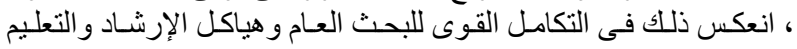

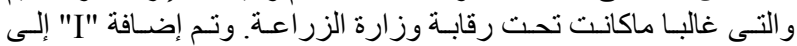

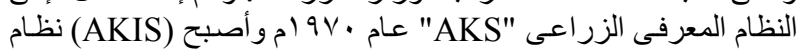

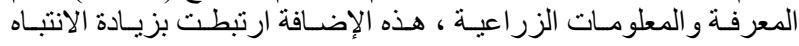

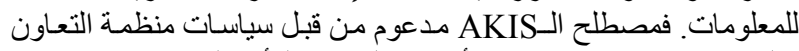

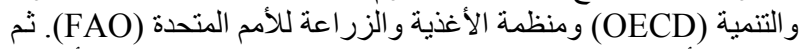

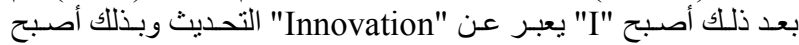
AKIS

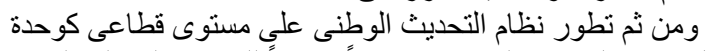

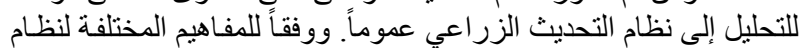

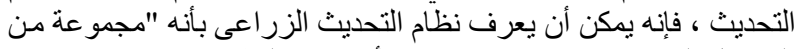

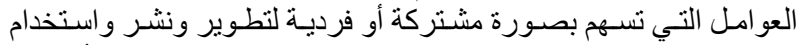

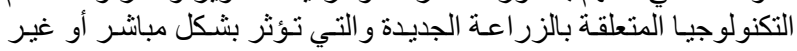

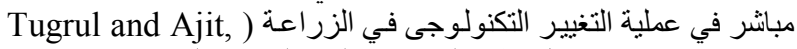

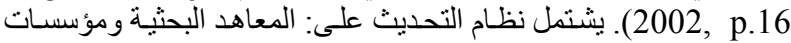

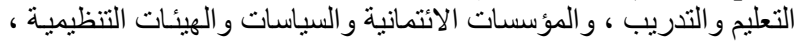

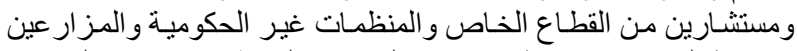

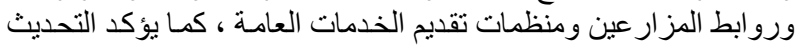

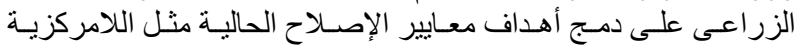

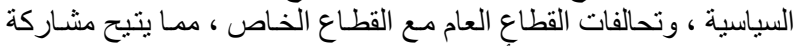

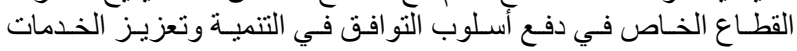

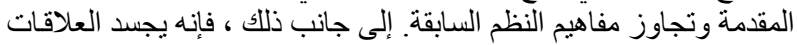

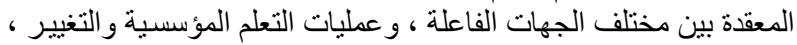

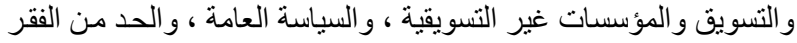

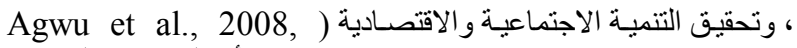

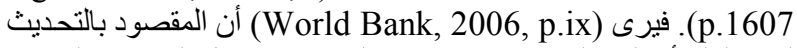

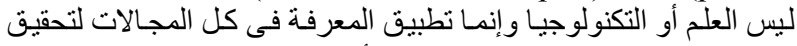

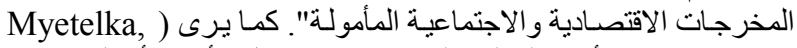

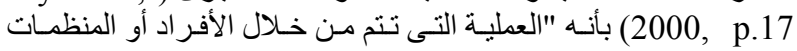

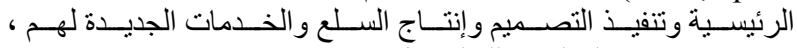

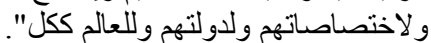

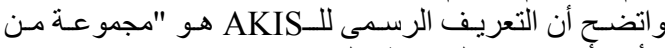

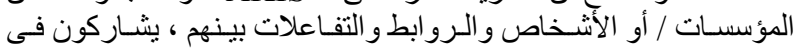

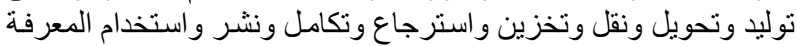

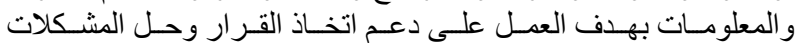

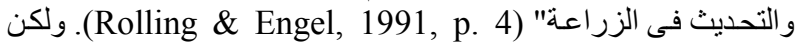

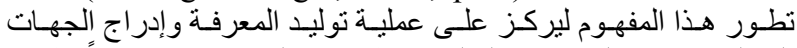

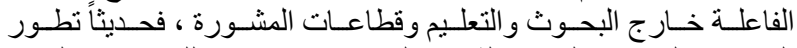
الـAKIS ليكتسب المعنى الثانى (التحديث) و الانفتاح للمزيـ من المناء المهام

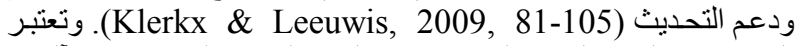

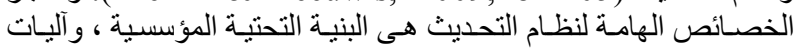
التمويل ، وخصـائص الثبكة و هيكل التسويق ( Klein-Woolthuis \&

.(et al., 2005, 609-619

كما أنشار (Euponou, 1993, p.86) إلى أن وظعائف نظام

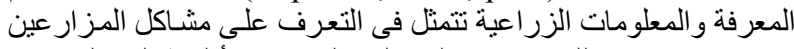

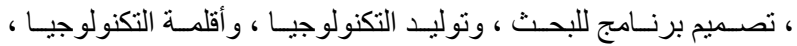
و استخدام التكنولوجيا وتنبيها ، وتقييم التكنولوجيا.

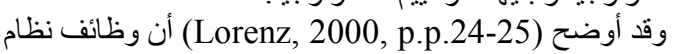

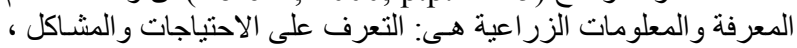

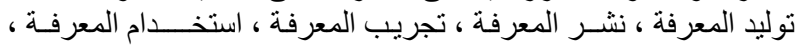

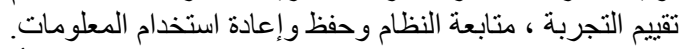

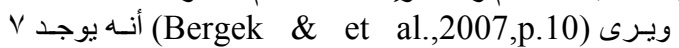

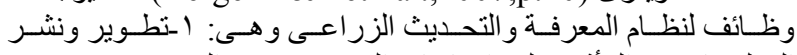

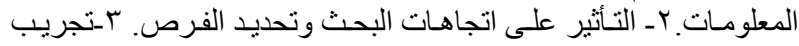

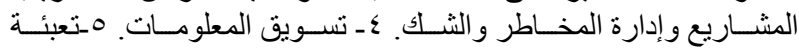

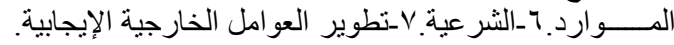




\section{J. Agric. Econom. and Social Sci,, Mansoura Univ., Vol.7 (1), january,2016}

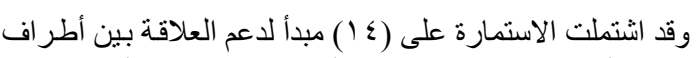

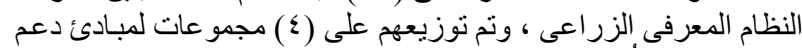

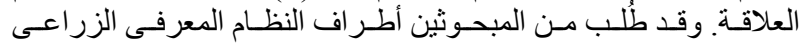

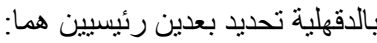

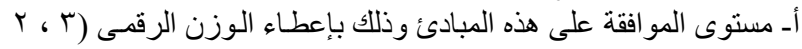

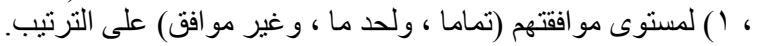

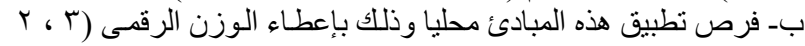

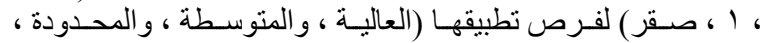
و المستحيلة) على الترتيب. هـ أدوات التحليل الإحصائي:

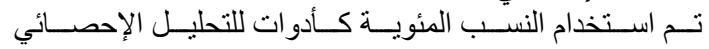
و استخلاص نتائج البحث.

\section{النتائج ومناقشتيها}

يعـرض هــا الجـز ء لنتـائج البحـث الميدانيـة ، و التـى يمكـن

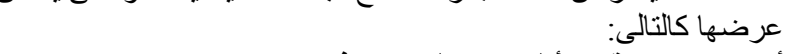

أولا : مستوى قبول أطراف النظام المعرفى الزراعى لمفترضـات التكامل

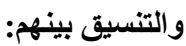

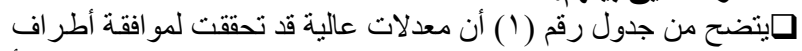

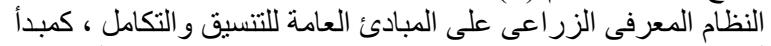

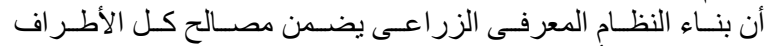

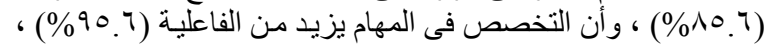

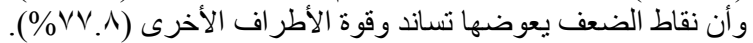

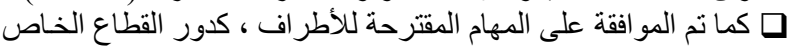

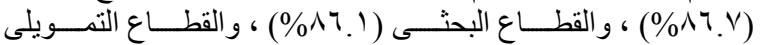

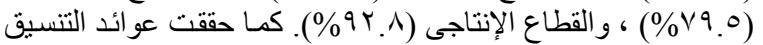

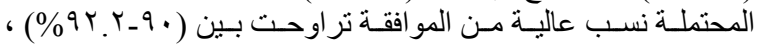

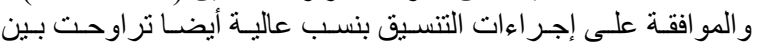

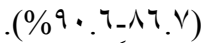

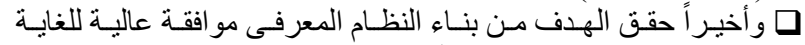

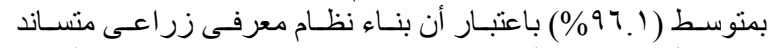

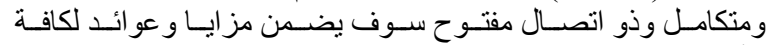
الأطر اف المشاركة.

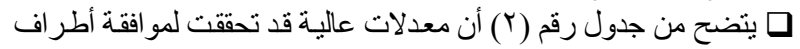

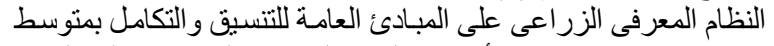

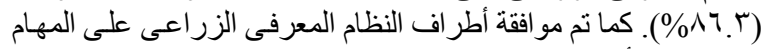

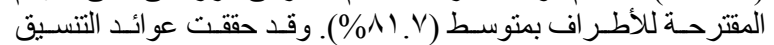

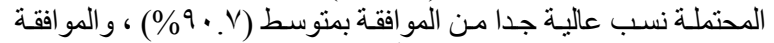

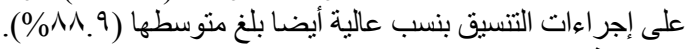

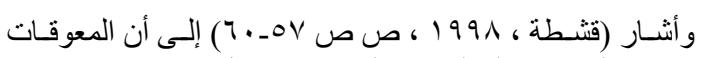

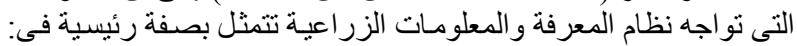

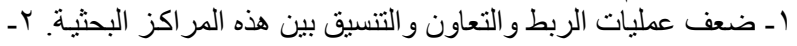

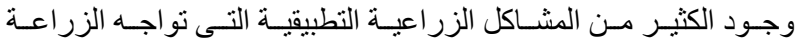

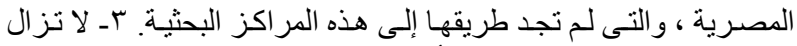

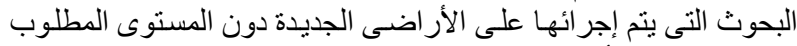

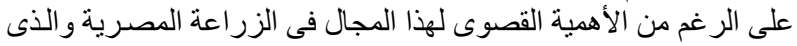

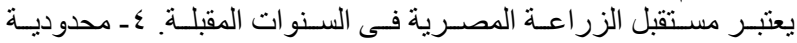

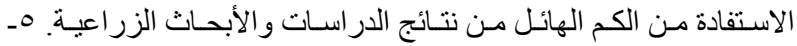

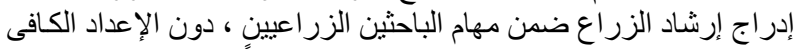

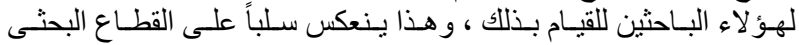

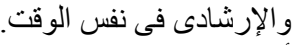
أهداف البحث

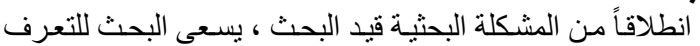

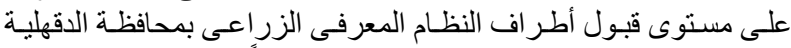
لمفترضات التكامل و التنسيق وفرص النطر تطبيقها محلياً.

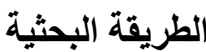

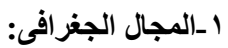

يقصد به المنطقة أو المناطق التى أجريت بها الدر اسة الميدانيـة وقد

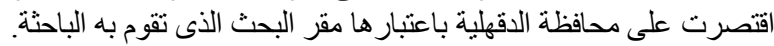

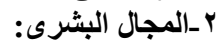

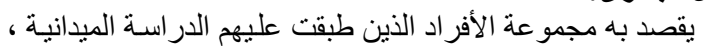

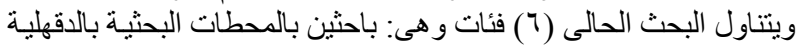

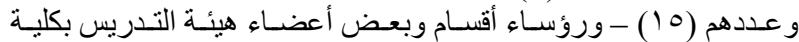

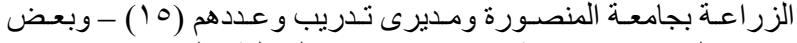

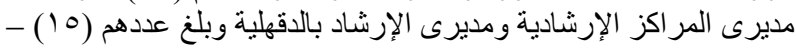

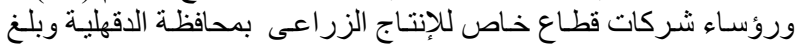

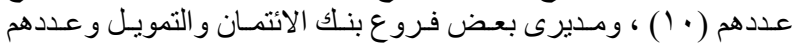

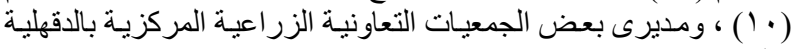
وبلغ عددهم (·) (1) ). (1).

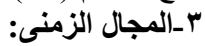

يقصد به الفترة الزمنية التى تم خلالها جمع البيانات للار اسـة

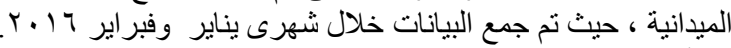
عـ أداة جمع البيانات:

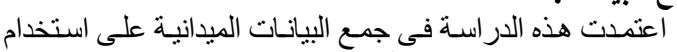

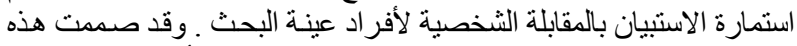
الاستمارة بحيث تقيس المتغير ات البحثية بما يتفق وتحقيق أهداف البحث.

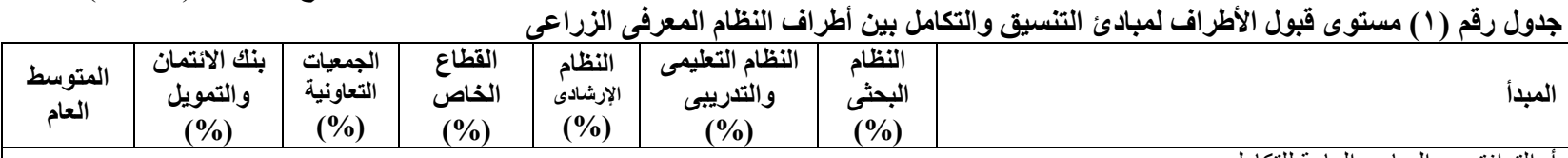

\begin{tabular}{|c|c|c|c|c|c|c|c|}
\hline \multicolumn{8}{|c|}{ 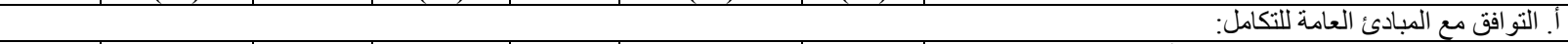 } \\
\hline 10.7 & 9. & A. & 9. & $1 \ldots$ & $9 \pi .4$ & 7. & 1 ـ بناء النظام المعرفى يضمن مصالح الأطر اف المشاركة \\
\hline 90.7 & $1 \cdots$ & A. & $1 \ldots$ & $1 \cdots$ & $1 \ldots$ & 94. & r _التخصص فى المهام يزيد من الكفاءة و الفعالية. \\
\hline VV.A & 7. & A. & $1 \ldots$ & $1 \cdots$ & N... & $\varepsilon \cdot$ & بـ ـ نقاط الضعف يعوضها تساند ونقاط قوة الأطر اف. \\
\hline \multicolumn{8}{|r|}{ ب. التو افق مع مهام أطر اف النظام المعرفى الزر اعى: } \\
\hline ᄉт.V & $1 \cdots$ & A. & $1 \cdots$ & ᄉ५. $\mathrm{V}$ & Aт.V & $77 . \mathrm{V}$ & ع ـ يختص القطاع الخاص بالمستلزمات و التسويق \\
\hline 1..1 & $1 \ldots$ & $\mathrm{ve}$ & $1 \ldots$ & A..V & N..V & VT.T & ه-يختص قطاع البحوث بتدريب الكو ادر ونشر المستحدثات \\
\hline 19.0 & $1 \ldots$ & v. & $1 \ldots$ & A. & A. & $\sum 7 . Y$ & آ ـ قطاع التمويل بتمويل المستلزمات واستثمار ات الانتاج \\
\hline 94.1 & $1 \cdots$ & $\mathrm{V} \cdot$ & $1 \cdots$ & ᄉт.V & $1 \cdots$ & $1 \cdots$ & V - يختص الزر اع و المنتجين بالعمليات الزر اعية الانتاجية \\
\hline \multicolumn{8}{|r|}{ ج. المو افقة مع إجراءات تكامل أطر اف النظام المعرفى الزر اعى: } \\
\hline Aт. $\mathrm{V}$ & $1 \cdots$ & A. & $\Lambda \cdot$ & $9 \pi .4$ & $9 \pi .4$ & VT.r & مـ بلورة صيغة للتعاقد وفقا لكل آلية بين كل طرفين. \\
\hline $9 \cdot .7$ & $1 \cdots$ & A. & 9. & 94.4 & 94.4 & ᄉт.V & 9-يتم تجريب هذه الصيغة فى منطقة جغر افية ( تجريب محدود) \\
\hline $19 . \varepsilon$ & $1 \cdots$ & A. & 9. & 94.4 & יז." & A. & • ا ـ تستخلص الدروس المستفادة وبلورة صيغة ملائمة \\
\hline \multicolumn{8}{|c|}{ د. المو افقة على عو ائد التنسيق بين أطر اف النظام المعرفى الزر اعى يضمن: } \\
\hline 9. & $1 \cdots$ & 9. & 9. & $1 \cdots$ & Rr.r & 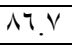 & 11 - زر اعة علمية و استخدام امكانات البحث العلمى. . \\
\hline $9 \cdot .7$ & $1 \cdots$ & 9. & $\wedge$. & $1 \cdots$ & A. & 94.4 & ب ا ـ تفر غ الزر اع والمنتجين للعطلية الانتاجية . \\
\hline 94.4 & $1 \cdots$ & 9. & 9. & $1 \cdots$ & $\wedge$. & 94.4 & r r ا_خلق طلب و اسع على خدمات القطاع الخاص التجارى \\
\hline 9. & $1 \cdots$ & 9. & 9. & $1 \cdots$ & A. & A. & ـ ا ـ تمويل مستدام للأنشطة و الاستثمار ات الزر اعية \\
\hline 97.1 & $1 \cdots$ & $9 \cdot$ & $1 \cdots$ & $1 \cdots$ & A५.V & $1 \cdots$ & الوصول لنظام معرفى متساند يضمن منافع كل الأطر اف \\
\hline
\end{tabular}


Zahran,Y. A. et al.

\begin{tabular}{|c|c|c|c|c|c|c|c|}
\hline المتوسط & $\begin{array}{c}\text { بنك الائتمان } \\
\text { والتمويل } \\
\text { (\%) }\end{array}$ & التعاونية & $\begin{array}{c}\text { الخطاص } \\
\text { (\%) }\end{array}$ & $\begin{array}{c}\text { الإرشادى النظام } \\
\text { (\%) }\end{array}$ & |النظام التعليمى & البحثى & 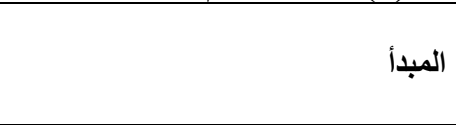 \\
\hline ᄉ५.r & NT.r & A. & $97 . V$ & $1 \cdots$ & 94.4 & $7 \varepsilon . \varepsilon$ & 1 ـ المبادئ العامة للتكامل. \\
\hline AI.V & $1 \cdots$ & VY.O & $1 \cdots$ & $\Lambda \cdot . r$ & $\wedge \wedge . \Sigma$ & ะ9.Y & ז ـ ـهام أطر اف النظام المعرفى الزر اعى. \\
\hline $9 \cdot . V$ & $1 \cdots$ & 9. & $\lambda V_{.0}$ & $1 \cdots$ & $\nabla \wedge . r$ & $\wedge \wedge . r$ & ــــإجر اءات تكامل أطر اف النظـام \\
\hline$\wedge \wedge .9$ & $1 \cdots$ & $\wedge$. & $\wedge \uparrow . \vee$ & 94.4 & 94.4 & $\Lambda$. & ــ المو افقة على عو ائد التنسيق بين الأطر اف. \\
\hline
\end{tabular}

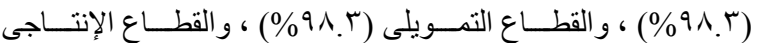

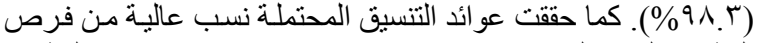

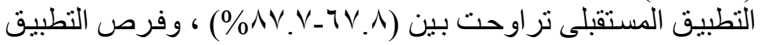

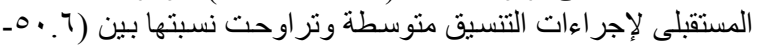
. \% \% Y .

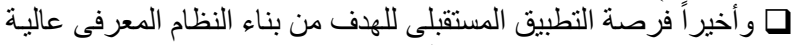

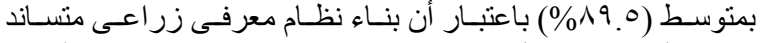

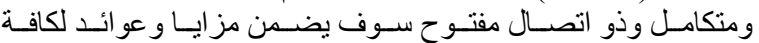

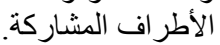

ثانيا: فرص التطبيق المستقبلى لمبادئ التكامل والتنسيق بين أطراف

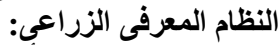

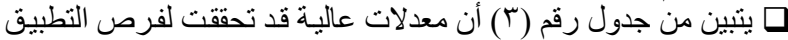

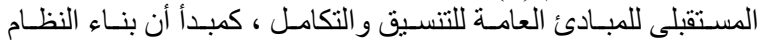

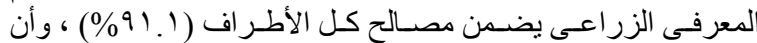

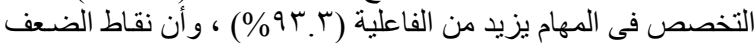

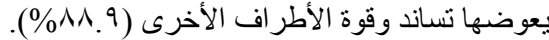

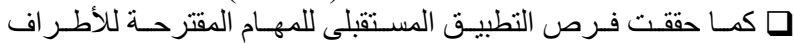

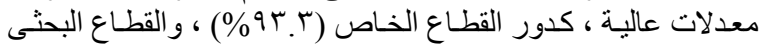

جدول رقم (r) فرص تطبيق مبادئ التنسيق والتكامل بين أطراف النظام المعرفى الزراعى مستقبلاً

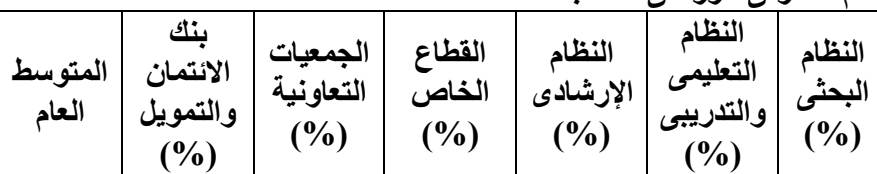

المـــبدأ

أ. التو افق مع المبادئ العامة للتكامل:

\begin{tabular}{|c|c|c|c|c|c|}
\hline 91.1 & $1 \ldots$ & $\wedge$. & $1 \ldots$ & $1 \ldots$ & ᄉ५. \\
\hline qr.r & $1 \ldots$ & $\Lambda$. & $1 \ldots$ & $1 \ldots$ & $1 \ldots$ \\
\hline $1 \wedge . q$ & $1 \ldots$ & $\Lambda$. & $1 \ldots$ & $1 \ldots$ & $1 \ldots$ \\
\hline
\end{tabular}

\begin{tabular}{|c|c|c|c|c|c|c|}
\hline $94 . r$ & $1 \ldots$ & 9. & $1 \ldots$ & $1 \ldots$ & $1 \ldots$ & \\
\hline $9 \wedge . r$ & $1 \ldots$ & 9. & $1 \ldots$ & $1 \ldots$ & $1 \ldots$ & 1 \\
\hline $9 \wedge . r$ & $1 \ldots$ & 9. & $1 \ldots$ & $1 \ldots$ & $1 \ldots$ & 1 \\
\hline $9 \wedge . r$ & $1 \ldots$ & 9. & $1 \ldots$ & $1 \ldots$ & $1 \ldots$ & 1 \\
\hline
\end{tabular}

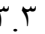

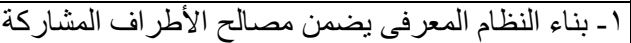

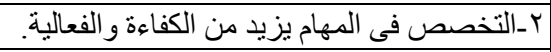

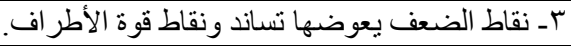

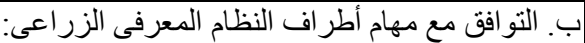

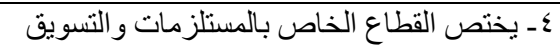

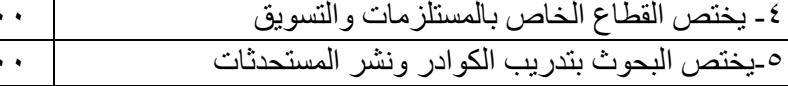

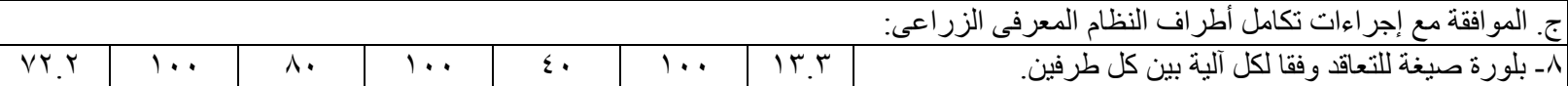

\begin{tabular}{|c|c|c|c|c|c|c|c|}
\hline 0.9 & r. & $\wedge$. & $1 \ldots$ & $\xi\rceil . \vee$ & $\varepsilon$. & 7.8 & 9-ينت تجريب هذه الصيغة فى منطقة جغر افية ( تجريب محدود) \\
\hline 77.1 & $r$. & 0. & $1 \ldots$ & $1 \ldots$ & $1 \ldots$ & YY.V & ملائمة \\
\hline
\end{tabular}

\begin{tabular}{|c|c|c|c|c|c|c|c|}
\hline & & & & & & \multicolumn{2}{|c|}{ د. المو افقة على عو ائد التنسيق بين أطر اف النظام المعرفى الزر اعى يضمن: } \\
\hline RT. & $1 \cdots$ & 7. & $1 \cdots$ & $\Lambda$. & or.r & $\leqslant 7.7$ & 11 ا - زر اعة علمية واستخدام امكانات البحث العلمى . \\
\hline AV.V & $1 \cdots$ & $\wedge$. & $1 \ldots$ & $\wedge \uparrow . \vee$ & $1 \ldots$ & 7. & r ا ـ تفرغ الزراع و المنتجين للعملية الانتاجية . \\
\hline VY.Y & $1 \cdots$ & $\wedge$. & $1 \ldots$ & RT.r & 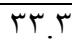 & $\varepsilon 7 . V$ & بر ا ـخلق طلب و اسع على خدمات القطاع الخاص التجارى \\
\hline$T \vee . \wedge$ & $1 \cdots$ & 7. & $1 \ldots$ & $77 . \mathrm{V}$ & $\varepsilon \cdot$ & $\varepsilon$. & ـ ـ ـ تمويل مستدام للأنشطة و الاستثمار ات الزر اعية . \\
\hline 19.0 & $1 \ldots$ & 9. & $1 \ldots$ & $1 \ldots$ & $94 . r$ & or. & الوصول لنظام معرفى متساند يضمن منافع كل الأطر اف \\
\hline
\end{tabular}

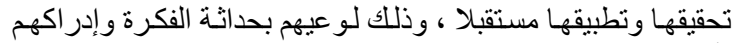

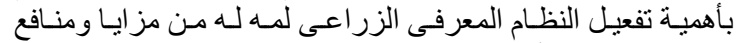
ستعود على كل الأطر اف. باف.

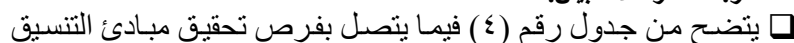

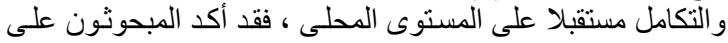

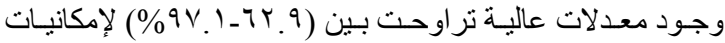

جدول (؛) ): استخلاص عام لفرص تطبيق مبادئ دعم العلاقة بين أطراف النظام المعرفى الزراعى محلياً

\begin{tabular}{|c|c|c|c|c|c|c|c|}
\hline المتوسط & $\begin{array}{c}\text { والتكو الائتمان } \\
\text { (\%) }\end{array}$ & التعمعيات & $\begin{array}{l}\text { الخاصاع) } \\
\text { (القاع) }\end{array}$ & $\begin{array}{c}\text { الإرشادى } \\
\text { النظام }\end{array}$ & 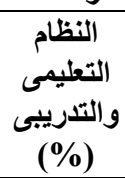 & $\begin{array}{l}\text { البخثى } \\
\text { النظى }\end{array}$ & 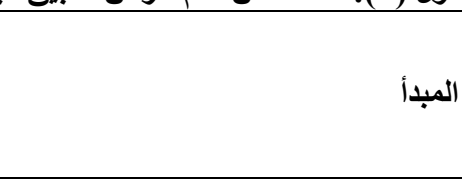 \\
\hline 91.1 & $1 \ldots$ & $\wedge$. & $1 \ldots$ & $1 \ldots$ & 90.7 & $\vee 1.1$ & 1 - المبادئ العامة للتكامل. \\
\hline $9 \vee .1$ & $1 \cdots$ & 9. & $1 \ldots$ & $1 \ldots$ & $1 \ldots$ & $1 \cdots$ & r - مهام أطر اف النظام المعرفى الزر اعى. \\
\hline 74.9 & 0 . & $\checkmark \cdot$ & $1 \ldots$ & TY.Y & $\wedge$. & 10.7 & ب-إجر اءات تكامل أطر اف النظام \\
\hline Vo.r & $1 \ldots$ & $\checkmark \cdot$ & $1 \ldots$ & $\vee \neg . V$ & $07 . \mathrm{V}$ & $\varepsilon \wedge . r$ & ـ ـ المو افقة على عو ائد التتسيق بين الأطر اف. \\
\hline
\end{tabular}


Eponou, T. (1993): Partners in Agricultural Technology: Linking Research and Technology Transfer to Serve Farmers, ISNAR Research Report, No.1, International Service for National Agricultural Research (ISNAR), Netherland: The Hague.

Klein-Woolthuis, R., Lankhuizen, M. and Gilsing, V. (2005) 'A system failure framework for innovation policy design', Technovation, Vol.25, 609-619.

Klerkx L. \& Leeuwis C. (2009): Shaping collective and Information Systems: The Positioning and Embedding of a Network Borker in the Dutch Dairy Sector, The Journal of Agricultural Education and Extension, 15(1).

Leeuwis, C. \& Van Den Ban, A. W. (2004): Communication for Rural Innovation: Rethinking Agricultural Extension, Oxford Blackwell Science Ltd. (3th ed.).

Lorenz, B. (2000): Review of Agricultural Knowledge System in Fiji: Opportunities and Limitations of Participatory Methods and Platforms tp Promote Innovation Development, Ph.D Dissertation, Berlin University, Germany.

Mytelka, Lynn K., (2000); "Local Systems of Innovation in a Globalized World Economy" in Industry and Innovation, Vol. 7. No. 1.

Reddy, E. R. (2008): Holistic View of Agricultural Information Transfer Systems, World Library and Information Congress: 74th IFLA General Conference and Council, 10-14 August, Québes, Canada, Available (http://www.ifla.org/IV/ifla74/index.htm)

Rivara, W. M. (2006): Agricultural Knowledge and Development in A New Age and a Different World, Association for International Agricultural and Extension Education (AIAEE), 13(2).

Röling, N. (1988): Extension Science: Information systems in Agricultural Development Cambridge University Press, UK.

Tugrul T, Ajit M (2002): The cotton supply chain in Azerbaijan, ISNAR, the Hague, Netherlands.

Van Den Ban, A. W. \& Hawkins, H. S. (1996): Agricultural Extension (2nd) ed., Blackwell Science, New York, U.S.A.

World Bank (2006): Enhancing Agricultural Innovation: How to Go Beyond the Strengthening of Research Systems: "Innovation System Capacity: A Comparative Analysis of Case Studies", Washington, DC.

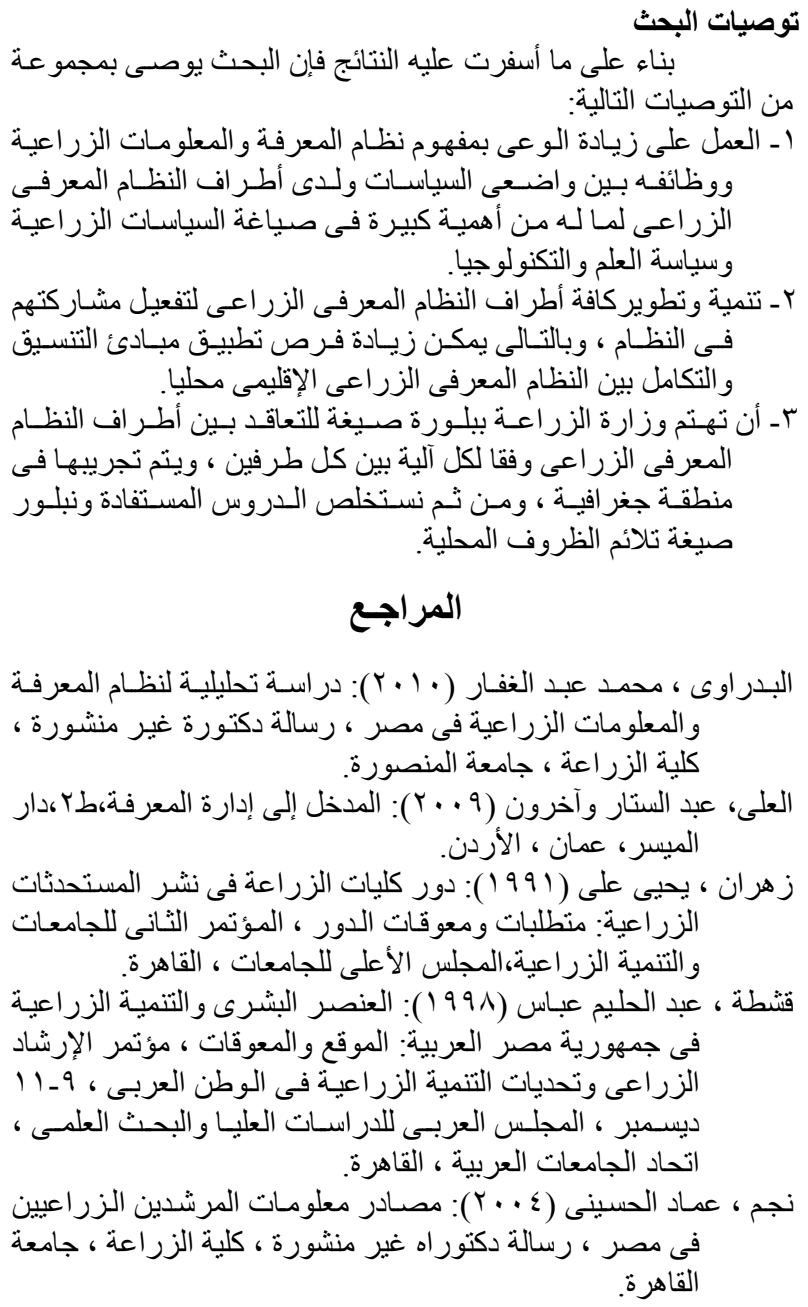

Agwu, A. E., Dimelu, M. U. \& Madukwe, M. C. (2008): Innovation system approach to agricultural development: Policy implications for agricultural extension delivery in Nigeria, African Journal of Biotechnology. 7 (11), 1604-1611.

Chema, S., Gilbert, E. \& Roseboom, L. (2003); A Review of Key Issues and Recent Experiences in Reforming Agricultural Research in Africa International Service for National Agricultural Research (ISNAR), Netherlands: The Hague. 


\title{
ACCEPTANCE AVAILABILITY OF AGRICULTURAL KNOWLEDGE SYSTEM ACTORS IN EL-DAKAHLIA GOVERNORATE FOR INTEGRATION AND COORDINATION ASSUMPTIONS \& ITS LOCAL APPLY OPPORTUNITIES \\ Zahran, Y. A..$^{(*)}$; E. Yousef ${ }^{(* *)} ;$ H. S. Kasem ${ }^{(*)}$ and Shimaa M. A. Naba ${ }^{(* *)}$ \\ ${ }^{(*)}$ Extension Agricultural and Rural Development Department, Faculty of Agric. - Mansoura University \\ ${ }^{(* *)}$ Extension Agricultural Researches Institute, Agricultural Researches center
}

\begin{abstract}
The Research aimed mainly to identify acceptance Availability of the Agricultural Knowledge system(AKS) Actors to Integration and Coordinatopn Assumption and its opportunities for Locally applied. The Sample of the research was selected from: (15) Researchers at Research stations in ElDakahlia, (15) heads of departments at Faculty of Agriculture-Mansoura University and managers of training, (15) managers of Extension Centers and and manager of Extension, (10) director of Private companies, (10) directors of Credit and Finance Bank's, (10) managers of Central cooperative Societies. The data was collected using a questionnaire method of personal interview during the period from January to February 2016. percentages was used as as a tool for statistical analysis and display search results.

The main results of the study were as follows :

- High rates was achieved on Acceptance of AKS Actors at the general principles of coordination and integration with average of $(86.3 \%)$. The AKS Actors accepted on Suggested tasks for actors with average $(81.7 \%)$. The Potential coordination returns also achieved very high rates of acceptance with average $(90.7 \%)$, and the acceptance of the coordination procedures at high rates also with average $(88.9 \%)$.

- The respondents confirmed the presence of high rates on opportunities achieving the principles of coordination and integration in the future at the local level, ranged between (62.9-97.1\%) for the potential achieving and applying them in the future.
\end{abstract}

